\section{PROTECTIVE CARE}

These measures are to be used for the patient's protection:

1. Private room.

2. Strict handwashing before patient care.

3. Care by employees free of infection.

4. Door closed at all times.

5. Stethoscopes and other shared equipment wiped off with alcohol before use

6. Double prepping of all needlestick and finger stick sites.

7. Limited visitors: only two at a time, free of infections please wash hands after entering the room.

Housekeeping:

1. Do not enter if you are sick. Call your supervisor.

2. Wear a yellow, disposable gown.

3. Wash hands and wear gloves.

4. Use freshly mixed cleaning solution, clean cloths and a fresh mop.

5. Wipe all surfaces, including:

Side rails

Telephone

Nurse-call bell

Bedside table

Television

Sink

6. Dispose of solution when you clean the toilet.

7. Mop the floor after removing all trash.

THANK YOU.
Figure.

sealed properly, I should think that there is no way to answer with certainty the question posed by Mr. Birnbaum.

My personal view, unsupported by a study, remains (Infection Control 1983; 4(1):9), that a three-month storage time would be entirely safe-assuming the package is not wet, damaged, or dropped on the floor.

George F. Mallison, MPH, PE Consultant, Environmental and Infection Control Glen Rock, New Jersey

\section{Software for Infection Control Data Gathering \\ To the Editor:}

Is there any software for Apple II Plus or TRS-80 Model 1 for infection control data gathering? I am aware of services that will compile this information using cards.

M.H. Moraleda, MD Chairperson

Infection Control Committee Veterans Administration Medical Center Battle Creek, Michigan
Donald L. Kaiser, DrPH, Associate Professor of Medicine, was invited to respond to Dr. Moraleda's query.

I am not aware of any software specifically for infection control data which will run on microcomputers of the size you mention (TRS-80, Apple II Plus). Our reporting systems require substantially larger machines (DEC PDP 11/24, 11/44, 11/70; DEC VAX 11/730, 11/750, 11/780). However, there are several excellent generalpurpose data base software packages (dBase II is a particularly flexible system) with which someone with moderate computer skills could construct a data system for infection control monitoring. The system would be limited by the available disk storage on the machine being used (though large-capacity hard disks are getting cheaper all the time), and would run relatively slowly (probably not an important problem).

I would advise an interested user to contact a software vendor and investigate, but take along someone who understands computers and your own needs to make sure that the system will suit your purposes.
Donald L. Kaiser, DrPH Associate Professor of Medicine Director, Clinical Computing Laboratory University of Virginia School of Medicine Charlottesville, Virginia

\section{"Reasonableness" in Kidney Transplant Precautions \\ To the Editor:}

We are involved in kidney transplants at our medical center and have really struggled to retain "reasonableness" in precautions with these patients. We have succeeded a bit in just getting the staff down to wearing only masks. What we would prefer is our Protective Care (Figure) which emphasizes handwashing.

I would appreciate your opinion on what is reasonable with transplant recipients.

\section{Sara L. Krantz, RN, BSN Hospital Epidemiologist Pitt County Memorial Hospital Greenville, North Carolina}

Sue Crow, RN, BSN, MSN, Associate Editor of Infection Control, was invited to respond to Ms. Krantz' letter.

Attempting reasonability in patient care procedures is somewhat perplexing in today's hospital. The initial response to any problematic patient care activity is "show me a study that proves. .." If research has been done in this area, data are reviewed, validity determined, and appropriate action taken. Unfortunately, very little research has been done in the infection control area. We cannot erroneously assume that since a particular area has not been studied there is not a problem in that area. The question then is how are decisions made without empirical data? Relying on timeproved principles of nursing may be the answer. Some people prefer to call this theoretical rationale-I call it common sense.

Recognizing this state of the art, let us review your guidelines for a com- 
promised host-the patient with a kidney transplant.

\section{PRIVATE ROOM}

Most infection control authorities agree that use of a private room does help in decreasing the risk of nosocomial infection by separating infected/colonized patients from the susceptible patient. A private room also acts as a reminder for personnel to wash their hands before leaving the room. Even though no studies document the validity of a private room, it makes good sense to use a private room for the compromised patient whenever possible.

\section{HANDWASHING}

Handwashing continues to be the most important means of preventing the spread of nosocomial infection. Enforcing this policy for the compromised patient is of the utmost importance. It makes good sense to use an antiseptic solution before direct contact with these patients. This provides assurance that the hands are as free from microorganisms as possible.

\section{EMPLOYEES FREE \\ FROM INFECTION}

Practical wisdom dictates that personnel do not work with patients who are sick and infected. This policy should be rigidly observed by all when working with compromised patients.

\section{CLOSING OF THE DOOR}

Why should the door be closed? Airborne contamination is not a primary method of nosocomial disease transmission. Furthermore, many patients develop claustrophobia when they are "shut-in" for long periods. Sometimes sound reasoning is not applied when establishing patient-care procedures; this is certainly the case of dictating that the door must be closed for the compromised patient.

\section{EQUIPMENT}

Equipment should be mechanically clean for all patients. This is especially true for such items that come into close contact with the patient (eg, stethoscopes). Although not a major source of contamination, such items should be wiped-down between each patient usage-again, common sense.

\section{SKIN PREPARATION}

Why a double skin prep? The bogus theory that, "if once does a little good, twice makes it even better" is being applied. There is absolutely no basis for this practice. This does not even make good sense and falls into the category of ritualistic thinking.

\section{VISITORS}

Visitors should check at the nurse's station before entering the compromised patient's room. This allows the nurse to make an assessment of their health and any other pertinent decisions. The number of visitors must be an individual assessment; there is nothing magical about only two visitors.

\section{HOUSEKEEPING}

Why do housekeeping personnel specifically need gowns and gloves? They, of all people, do not need extra frock because they do not come into direct contact with the patient. (The lack of judgment sometimes used is atrocious.) Other housekeeping activities mentioned in your letter should be carried out during any housekeeping activity and not be limited to the compromised patient.

Studies show that conventional protective isolation does not affect the nosocomial infection rate of the compromised patient. ${ }^{1}$ Therefore, most hospitals have discarded the concept of protective isolation. At our hospital, we feel that hospital personnel need a reminder that certain patients are compromised, therefore, we place a sign on the door to remind personnel to wash their hands and not to enter if they have any type of infection.

Protecting any patient from nosocomial infection is a challenge in today's world. Obviously more studies need to be done in specific patient care activities so that more empirical data can be used to make recommendations. Until then, basic nursing principles and common sense must be employed to protect the patient.

I wish you success in using the common sense approach. It is challenging but can be successful.

\section{REFERENCES}

1. Nauseef WM, Maki DG: A study of the value of simple protective isolation in patients with granulocytopenia. N Engl J Med 304(8): 448-455.
Sue Crow, RN, BSN, MSN

Associate Editor

Infection Control

\section{Age Distribution of Patients with Nosocomial Infections}

\section{To the Editor:}

The data presented by Gross et al of Infection Control, in "Nosocomial Infections: Decade-Specific Risk" in the May/June 1983 issue confirmed our study, "Analysis of the Age Distributions of Patients with Nosocomial Infections in the Community Hospital," presented at both the Pennsylvania Infection Control Symposium in October 1981, and the 1982 APIC Meeting in New Orleans.

After analyzing our nosocomial infection data in ten-year age ranges for three years (1978 to 1980) we also demonstrated that in the age groups from ages 61 to 90 , the percentage of infections was approximately two times higher than admissions. A chisquare test for Goodness of Fit was determined in the study to show a highly significant difference within the age distribution (1978 to $1980 \times$ $2 / 9=516.81, \alpha=0.005)$. We also showed that $68.8 \%$ of UTIs, $62.8 \%$ of respiratory infections, $52.2 \%$ of wound infections, $55.9 \%$ of blood, and $33.3 \%$ of other types of nosocomial infections occurred in the population 61 years old or older. Patients 61 years old or older were responsible for $59.9 \%$ of all nosocomial infections while this group accounted for $29.4 \%$ of all admissions.

With the confirmation of our data by Gross and co-workers, I would suggest that all hospitals consider the elderly patient at increased risk and evaluate their policies and procedures to increase the quality of care given to the 60-year and above age range.

Loretta Fauerbach, MS Director of Epidemiology The Williamsport Hospital Williamsport, Pennsylvania 